\title{
Tolérance de quelques accessions de patate douce (Ipomoea batatas L.) cultivées au sud-Bénin aux charançons de la patate douce (Cylas spp.) sous infestation naturelle
}

\author{
*Régina KOTCHOFA, André FANOU, Hugues BAIMEY, Florentin AZANMA, Lionel ZADJI, Epiphane \\ SODJINOU \\ Faculté d'Agronomie, Université de Parakou, BP 123, Route de l'Okpara, Parakou, Bénin \\ Adresse de correspondance : email: *kotchofaregina@yahoo.fr ; Tel : (+229) 97635167 \\ Original submitted in on 24th July 2019. Published online at www.m.elewa.org/journals/ on $31^{\text {st }}$ August 2019 \\ https://dx.doi.org/10.4314/jab.v140i1.8
}

\section{RÉSUMÉ}

Objectif: Identifier les accessions indigènes de patate douce les plus tolérantes aux charançons de la patate douce, Cylas spp., en milieu réel.

Méthodologie et résultats: L'essai a été réalisé avec 42 accessions de patate douce dans un champ paysan à forte infestation de Cylas spp., au sud-Bénin. Un dispositif en blocs incomplets avec six répétitions a été utilisé. Neuf groupes d'accessions ont été identifiés. Le plus faible taux d'infestation $(0,00 \pm 0,00 \%)$ des racines tubéreuses a été enregistré dans le groupe 4 ayant un péricarpe et une pulpe de couleur orange et le plus faible rendement $(2,40 \pm 1,71 \mathrm{t} / \mathrm{ha})$. Le plus fort taux d'infestation $(36,74 \pm 6,24 \%)$ a été enregistré dans le groupe 8 ayant un péricarpe et une pulpe de couleur crème et un rendement moyen $(6,79 \pm 0,73 \mathrm{t} / \mathrm{ha})$. Le plus haut rendement $(10,31 \pm 0,97 \mathrm{t} / \mathrm{ha})$ et le poids moyen des racines tubéreuses le plus élevé $(443,20 \pm 53,30 \mathrm{~g})$ ont été obtenus dans le groupe 6 ayant le plus faible nombre de racines tubéreuses par plant $(1,00 \pm 0,00)$. Les accessions du groupe 2 ont donné le plus grand nombre de racines tubéreuses par plant $(2,77 \pm 0,43)$. Le plus faible poids moyen des racines tubéreuses $(133,33 \pm 42,49 \mathrm{~g})$ a été enregistré dans le groupe 9.

Conclusion et application des résultats: Les accessions du groupe 4 semblent intéressantes pour un programme de lutte contre Cylas spp. Celles des groupes 2 et 6 présentant de très bons paramètres agronomiques pourraient être utilisées comme "parents» dans un programme de sélection et d'améliorations variétales pour soutenir les performances des accessions du groupe 4. La lutte variétale contre Cylas spp. peut être menée au champ en utilisant l'accession V37, seule accession de patate douce appartenant au groupe 4 mais tout en l'améliorant génétiquement pour de plus grandes productions.

Mots clés : Accessions indigènes de patate douce, infestation naturelle, Cylas spp., paramètres agromorphologiques, tolérance, sud-Bénin. 
Kotchofa et al., J. Appl. Biosci. 2019 Tolérance de quelques accessions de patate douce (Ipomoea batatas L.) cultivées au sud-Bénin aux charançons de la patate douce (Cylas spp.) sous infestation naturelle

Tolerance of some sweet potato (Ipomoea batatas L.) accessions cultivated in southern Benin to sweet potato weevils (Cylas spp.) under natural infestation

\section{ABSTRACT}

Objectives: To Identify the most tolerant indigenous sweet potato accessions to the sweet potato weevils, Cylas spp. in the natural environment.

Methodology and Results: The experiment was conducted with 42 sweet potato accessions in a farmer field with a heavy infestation of Cylas spp. in southern Benin. An incomplete block design with six repetitions was used. Nine groups of accessions were identified. The lowest infestation rate $(0.00 \pm 0.00 \%)$ of tuberous roots was recorded in group 4 with an orange pericarp and pulp and the lowest yield $(2.40 \pm 1.71 \mathrm{t} / \mathrm{ha})$. The highest infestation rate $(36.74 \pm 6.24 \%)$ was recorded in group 8 with a creamy pericarp and pulp and a mean yield $(6.79 \pm 0.73 \mathrm{t} / \mathrm{ha})$. The highest yield $(10.31 \pm 0.97 \mathrm{t} / \mathrm{ha})$ and the highest tuberous root weight $(443.20 \pm 53.30 \mathrm{~g})$ were obtained in group 6 with the lowest tuberous root number $(1.00 \pm 0.00)$ per plant. Group 2 accessions, however, yielded the highest tuberous root number $(2.77 \pm 0.43)$ per plant. The lowest tuberous root weight $(133.33 \pm 42.49 \mathrm{~g})$ was recorded in group 9 .

Conclusions and application of findings: Sweet potato accessions of group 4 seem interesting for a control program against Cylas spp. Sweet potato accessions of groups 2 and 6 with very good agronomic parameters could be used as "parents" in a selection and varietal improvement program to support the performance of group 4 accessions. The varietal control against Cylas spp. can be done in the field using accession V37, the unique accession of sweet potato belonging to group 4, but by improving it genetically for highest yields.

Key words: Indigenous accessions of sweet potato, natural infestation, Cylas spp., agro-morphological parameters, tolerance, southern Benin.

\section{INTRODUCTION}

Au Bénin, la patate douce (PD, Ipomoea batatas L.) occupe le troisième rang parmi les racines et tubercules après l'igname et le manioc avec une production nationale estimée à 58.145 tonnes en 2016 (FAOSTAT, 2017). Malheureusement, les charançons de la PD, Cylas spp. sont une contrainte majeure à sa production et à son utilisation dans le pays (Baimey et al., 2017). En Afrique, C. puncticollis Boheman et C. brunneus Fabricius sont les espèces de charançons de PD les plus répandues (Andrade et al., 2009) occasionnant des pertes de rendement atteignant $100 \%$ particulièrement en saison sèche et dans les stocks (Smit et al., 2001). Aussi, même les faibles niveaux d'infestation de ces ravageurs réduisent-ils considérablement la qualité gustative et organoleptique des racines tubéreuses (Stathers et al., 2003). En effet, le stade le plus dommageable de l'insecte est la larve (CIP, 2013 ; Stathers et al., 2013). Elle se nourrit à l'intérieur des racines tubéreuses en creusant des galeries qui provoquent un dessèchement de l'intérieur faisant perdre le poids aux racines tubéreuses (Okonya et Kroschel, 2013). Selon Starthers et al. (2013), ces galeries constituent aussi des points d'entrée à d'autres organismes nuisibles tels que les bactéries, les champignons ou les virus. En réponse à la perturbation engendrée par la présence de l'insecte, les racines tubéreuses produisent des composés (terpénoïdes) qui leur confèrent un goût amer et une odeur nauséabonde, les rendant ainsi non propices à la consommation et réduisant leur valeur marchande (Varin et al., 2009). Des méthodes efficaces de gestion durable de ces ravageurs peuvent donc permettre un accroissement significatif de la production de la PD et une amélioration du niveau de vie de milliers de pauvres exploitants agricoles à travers l'Afrique sub-saharienne. Une approche plausible de lutte contre ce ravageur est l'utilisation des variétés résistantes qui est considérée selon Muyinga et al. (2012) comme une stratégie de lutte économique et efficace contre Cylas spp. Des 
progrès significatifs ont été réalisés dans l'identification de la tolérance de la PD à Cylas spp. Des niveaux acceptables de tolérance à l'insecte ont été récemment identifiés chez des accessions de PD (Jackson et al., 2012) dans certains pays tels que la Tanzanie (Kagimbo et al., 2017) et les Etats-Unis (Jackson et al., 2012). Au Bénin, malgré les travaux réalisés sur la PD

\section{MATÉRIEL ET MÉTHODES \\ Matériel}

Site expérimental. L'essai a été réalisé de juin à novembre 2016 et 2017 dans un champ paysan (N06³3'06.6" ; E001 '50'28.7" ; 65 m d'altitude) de PD situé à Tohon, un village de la commune de Houéyogbé au sud-Bénin. Ce champ a été retenu comme l'un des sites les plus infectés par Cylas spp. Iors des prospections effectuées en novembre-décembre 2015 sur la distribution du ravageur dans les champs de PD au Sud-Bénin. Le pourcentage d'infection des racines tubéreuses du champ retenu a été évalué à $35 \%$.

Matériel végétal. Quarante-deux accessions de $\mathrm{PD}$ (Tableau 1) dans l'essai. Les accessions ont été collectées au cours des prospections ci-dessus indiquées. Elles ont été ensuite multipliées sur un site non infecté par Cylas spp. Des boutures ont été prélevées de ce site pour l'essai à Tohon.

Méthodes

Installation de l'essai: Un dispositif en blocs incomplet avec six répétitions a été utilisé. Chaque répétition correspondait à un bloc composé de quatre
(Baimey et al., 2017 ; Agalè, 2016), l'étude du développement de variétés de PD résistantes à Cylas spp. nécessite d'être faite. La présente étude vise à identifier en milieu paysan des accessions indigènes de PD tolérantes à Cylas spp. et susceptibles d'être proposées dans des programmes de sélection et d'amélioration variétales.

sous-blocs incomplets. Deux sous-blocs comportaient chacun une ligne de dix buttes de $1 \mathrm{~m}$ de hauteur chacune et les deux autres sous-blocs comportaient chacun une ligne de onze buttes. Des écartements de $1 \mathrm{~m}$ et de 1,5 m ont été observés entre les sous-blocs et les blocs, respectivement. Par contre, entre deux buttes consécutives, un écartement de 0,80 m a été observé. Au total 42 buttes à raison d'une butte par accession, ont été confectionnées par bloc. Des boutures des différentes accessions de PD (d'environ 30 à $40 \mathrm{~cm}$ de longueur comportant chacune quatre à sept nœuds selon l'accession) ont été utilisées pour la plantation. Les boutures ont été individuellement plantées de façon horizontale dans les buttes à une profondeur d'environ 10 à $15 \mathrm{~cm}$ avec les deux extrémités libres. Deux sarclages ont été faits un et trois mois après plantation, respectivement. Aucun fertilisant ni pesticide n'a été utilisé durant toute la phase de l'essai. La récolte a été faite manuellement cinq mois après la plantation à cause de la variabilité des accessions.

Tableau 1 : Accessions de patate douce utilisées pour l'essai et leur provenance

\begin{tabular}{|c|c|c|}
\hline \multirow[t]{2}{*}{ Accession de patate douce } & \multicolumn{2}{|c|}{ Provenance } \\
\hline & Commune/ Département & $\begin{array}{l}\text { Zone agro- } \\
\text { écologique* }\end{array}$ \\
\hline $\begin{array}{l}\text { V4, V5, V8, V9, V14, V27, V28 } \\
\text { V1A, V1B, V15A, V15B, V21, V24 } \\
\text { V11, V12, V16, V18, V19, V22, V23, V26, V30A, } \\
\text { V30B, V31, V32, V33, V34, V35, V36, V37, V38 } \\
\text { V39 } \\
\text { V40, V41 } \\
\text { V42, V43, V44 } \\
\text { V47, V49 } \\
\text { V48 } \\
\text { V10 } \\
\text { V45 }\end{array}$ & $\begin{array}{l}\text { Sakété / Plateau } \\
\text { Ifangni / Plateau } \\
\text { Adjohoun / Ouémé } \\
\text { Adja-Ouèrè / Plateau } \\
\text { Comè / Mono } \\
\text { Houeyogbé / Mono } \\
\text { Dogbo / Couffo } \\
\text { Toviklin / Couffo } \\
\text { Dangbo / Ouémé } \\
\text { Bopa / Mono }\end{array}$ & $\begin{array}{l}6 \\
6 \\
8 \\
7 \\
8 \\
6 \\
6 \\
6 \\
8 \\
6\end{array}$ \\
\hline
\end{tabular}

${ }^{*} 6,7$ et $8=$, respectivement, Zone agro-écologique 6 (Zone de terre de barre), Zone 7 (Zone de la dépression) et Zone 8 (Zone des pêcheries) (DPP-MAEP, 2001) 
Collecte de données : Les données ont été collectées dans trois blocs en laissant les blocs situés aux extrémités du site expérimental. Trois évaluations à intervalles d'un mois ont été faites à la phase végétative et une évaluation à la récolte sur toutes les accessions de PD dans les trois blocs retenus. Un descripteur de la PD (CIP/AVRDC/IBPGR, 1991) a été utilisé pour évaluer des paramètres sur les accessions plantées. A la phase végétative, les paramètres: couverture du sol par la plante (CS), longueur des entre-nœuds (EN), longueur des pétioles (LP), longueur de la tige principale (LT), dimension des feuilles étalées (DF), aspect général des feuilles (AGF), type de lobation ( $\mathrm{TL}$ ), nombre de lobes (NL), forme du lobe central du limbe (FL), couleur des feuilles matures (CFM), couleur des feuilles immatures (CFI), couleur prédominante de la tige principale (CPT), couleur secondaire de la tige principale (CST), pigmentation du pétiole (PP), pubescence du sommet de la tige (Pub) ; ont été évalués. A la récolte, les paramètres suivants ont été évalués : arrangement des racines tubéreuses dans le sol (ARTS); longueur de la tige joignant les racines tubéreuses à la tige principale (LTRTTP); imperfections de surface des racines tubéreuses (ISRT), fêlures sur les racines tubéreuses (FRT), forme des racines tubéreuses (FRT), épaisseur du péricarpe des racines tubéreuses (EPRT), couleur prédominante du péricarpe des racines tubéreuses (CPPRT), intensité de la couleur prédominante du péricarpe des racines tubéreuses (ICPRT), couleur secondaire du péricarpe des racines tubéreuses (CSPRT), couleur prédominante de la pulpe des racines tubéreuses (CPPuRT), couleur secondaire de la pulpe des racines tubéreuses (CSPuRT), distribution de la couleur secondaire de la pulpe des racines tubéreuses (DCSPuRT), variation de la forme des racines tubéreuses (VFRT), variation de la taille des racines tubéreuses (VTRT), production de latex par les racines tubéreuses (PLRT), oxydation des racines tubéreuses (ORT), longueur des racines tubéreuses (LgRT), diamètre des racines tubéreuses (DRT), poids des racines tubéreuses (PRT), nombre de racines

\section{RÉSULTATS ET DISCUSSION}

Variabilité morphologique des accessions de patate douce : Une variabilité a été enregistrée au sein des différents paramètres morphologiques étudiés. La CHA a permis d'obtenir des groupes d'accessions de PD constitués sur la base de la variabilité morphologique obtenue pour les paramètres qui se sont révélés fortement corrélés ( $r>0,59$ au seuil de 1\%). II s'agit tubéreuses (NbRT) par plant et biomasse aérienne des feuilles et tiges (Bma). Le rapport DRT/LgRT a permis de voir si les racines tubéreuses présentent une forme ronde car elles le sont, selon Tarini et al. (2007), lorsque la valeur du rapport est proche de 0,5. Pour évaluer la tolérance des accessions à Cylas spp., le nombre de racines tubéreuses endommagées par l'insecte et le degré d'infestation sur les racines tubéreuses ont été évalués visuellement. Le degré d'infestation a été apprécié suivant l'échelle de Starthers et al. (2003) où $1=0 \%$ (aucune infestation), 2 $=1-25 \%$ (très faible infestation), $3=26-50 \%$ (infestation modérée), $4=51-75 \%$ (infestation considérable) et $5=$ 76-100\% (infestation sévère).

Le pourcentage de racines tubéreuses infestées par Cylas spp. (PI) a été calculé suivant la formule décrite par Bassey (2012) :

$\mathrm{PI}=$ (Nombre de racines tubéreuses endommagées/nombre total de racines tubéreuses) $\mathrm{x}$ 100 ,

L'indice de sévérité (IS) des dégâts causés par Cylas spp. a été quant à lui calculé suivant la formule décrite par Jackson et al. (2012) :

IS $=[(a x$ échelle1 $)+(a x$ échelle2 $)+\ldots+(a x$ échelle5)] / nombre total de racines tubéreuses

Où « a » est le nombre de racines tubéreuses dans la classe d'échelle. L'extrême supérieur de chaque échelle a été utilisé pour le calcul de l'indice de sévérité.

Analyse de données: Les données liées aux paramètres morphologiques ont été d'abord soumises à une analyse de corrélation pour identifier les paramètres explicatifs de la diversité morphologique. Ces données ont été ensuite soumises à une classification hiérarchique ascendante (CHA) avec comme paramètre la distance euclidienne et la méthode d'agrégation Ward en vue de regrouper les accessions. Les autres données ont été soumises à une analyse de variance. Le test de classification multiple de Duncan a permis de séparer les moyennes au seuil de 5\%. Le logiciel SAS version 9.2 a été utilisé pour analyser les données.

de : AGF, TL, NL, FL, CPT, CST, CPPRT, CPPuRT, CSPuRT et DCSPuRT. Le dendrogramme (Figure 1) obtenu à partir de la $\mathrm{CHA}$ a séparé les 42 accessions de PD étudiées en neuf différents groupes avec une distance euclidienne s'étendant de 0 à 5 . Le Tableau 2 présente la composition et les caractéristiques des groupes obtenus sur la base des paramètres qui se 
sont révélés fortement corrélés. Des études réalisées sur la PD au Burkina Faso (Koussao et al., 2014), au Ghana (Amoatey et al., 2016) et au Kenya (Karuri et al.,
2010) ont également montré des variabilités morphologiques liées au TL, à la CPPRT et à d'autres paramètres tels l'AGF et la FL des feuilles.

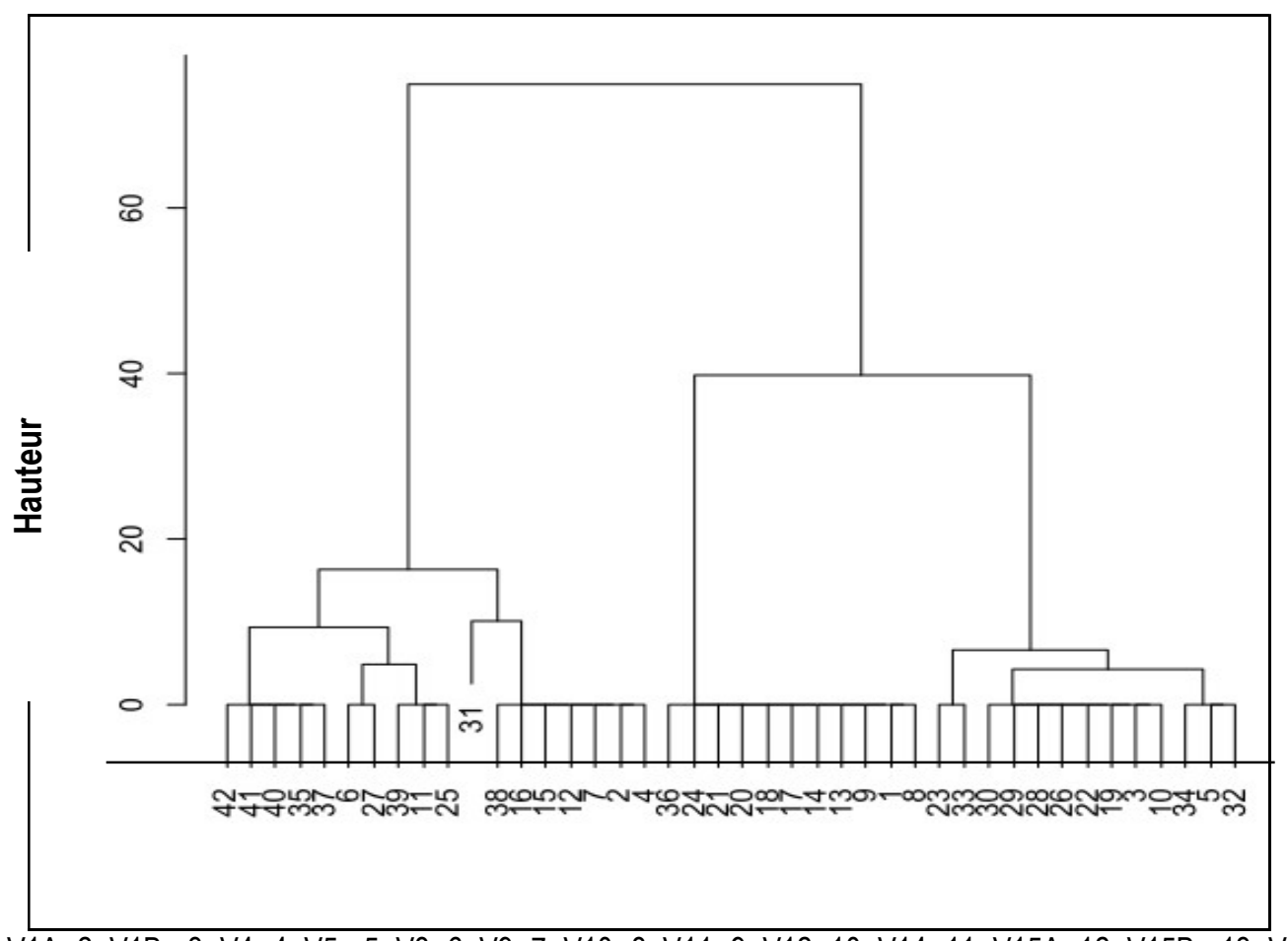

Légende: 1=V1A; 2=V1B ; 3=V4; 4=V5 ; 5=V8; 6=V9; 7=V10; 8=V11; 9=V12; 10=V14; 11=V15A; 12=V15B; 13=V16; $14=\mathrm{V} 18$ $15=\mathrm{V} 19 ; 16=\mathrm{V} 21 ; 17=\mathrm{V} 22 ; 18=\mathrm{V} 23 ; 19=\mathrm{V} 24 ; 20=\mathrm{V} 26 ; 21=\mathrm{V} 27 ; 22=\mathrm{V} 28 ; 23=\mathrm{V} 29 ; 24=\mathrm{V} 30 ; 25=\mathrm{V} 31 ; 26=\mathrm{V} 32 ; 27=\mathrm{V} 33 ; 28=\mathrm{V} 34 ;$ $29=\mathrm{V} 35 ; 30=\mathrm{V} 36 ; 31=\mathrm{V} 37 ; 32=\mathrm{V} 38 ; 33=\mathrm{V} 39 ; 34=\mathrm{V} 40 ; 35=\mathrm{V} 41 ; 36=\mathrm{V} 42 ; 37=\mathrm{V} 43 ; 38=\mathrm{V} 44 ; 39=\mathrm{V} 45 ; 40=\mathrm{V} 47 ; 41=\mathrm{V} 48 ; 42=\mathrm{V} 49$

Figure 1: Dendrogramme issu de la classification hiérarchique ascendante de 42 accessions de patate douce évaluées. Les groupes des accessions de patate douce obtenus sont en abscisse.

Tableau 2 : Composition et quelques caractéristiques des groupes d'accessions de patate douce évaluées

\begin{tabular}{|c|c|c|c|c|c|}
\hline Groupe & $\begin{array}{c}\text { Nombre } \\
\text { d'accessions } \\
\text { de PD }\end{array}$ & Composition des groupes & $\begin{array}{l}\text { Type de lobation } \\
\text { des feuilles }\end{array}$ & $\begin{array}{c}\text { Couleur } \\
\text { péricarpe des } \\
\text { RT }\end{array}$ & $\begin{array}{c}\text { Couleur } \\
\text { pulpe des } \\
\text { RT }\end{array}$ \\
\hline 1 & 05 & V41, V43, V47, V48, V49 & Modérément & Rouge pourpre & Blanc \\
\hline 2 & 02 & V9, V33 & Légèrement & Rouge pourpre & $\begin{array}{l}\text { Crème- } \\
\text { foncé }\end{array}$ \\
\hline 3 & 03 & V15A, V31, V45 & Légèrement & Rouge pourpre & Blanc \\
\hline 4 & 01 & V37 & Modérément & Orange & Orange \\
\hline 5 & 07 & $\begin{array}{l}\text { V1B, V5, V10, V15B, V19, V21 } \\
\text { et V44 }\end{array}$ & Légèrement & Rose & Crème \\
\hline 6 & 11 & $\begin{array}{l}\text { V1A,V11,V12,V16,V18,V22,V23, } \\
\text { V26,V27,V30A,V42 }\end{array}$ & Légèrement & Crème & Crème \\
\hline 7 & 02 & V30B et V39 & Modérément & Crème & Blanc \\
\hline 8 & 08 & $\begin{array}{l}\text { V4, V14, V24, V28, V32, V34, } \\
\text { V35, V36 }\end{array}$ & Profondément & Crème & Crème \\
\hline 9 & 03 & V8, V38, V40 & Profondément & Crème & Blanc \\
\hline
\end{tabular}

$\mathrm{RT}=$ racines tubéreuses, $\mathrm{PD}=$ patate douce. 
Paramètres agronomiques des accessions de patate douce : Le Tableau 3 montre des différences très hautement significatives $(P<0,001)$ observées entre les groupes d'accessions de PD pour la LgRT, le Rend et le DRT/LgRT. Le rendement (Rend) en racines tubéreuses a varié entre les différents groupes d'accessions de PD. Le plus haut rendement $(10,31 \pm 0,97 \mathrm{t} / \mathrm{ha})$ a été obtenu au niveau des accessions du groupe 6 tandis le rendement le plus faible a été enregistré au niveau des accessions du

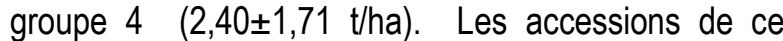
groupe ont également donné le plus faible $\mathrm{NbRT}$ par plant $(1,00 \pm 0,00)$ tandis que les accessions du groupe 2 ont donné le plus fort NbRT par plant $(2,77 \pm 0,43)$. Le PmRT des accessions du groupe 6 a été le plus élevé $(443,20 \pm 53,30)$ contre $133,33 \pm 42,49$ au niveau des accessions du groupe 9. La plus grande Bma $(14,20 \pm 2,96)$ a été celle des accessions du groupe 7 tandis que la plus faible Bma $(5,72 \pm 1,63)$ a été enregistrée au niveau des accessions du groupe 4. Les accessions du groupe 8 ont présenté la plus grande $\operatorname{LgRT}(19,83 \pm 1,01)$ tandis que celles du groupe 4 ont présenté la plus faible longueur $(12,50 \pm 1,50)$. Le DRT le plus élevé $(8,36 \pm 0,86)$ a été celui des accessions du groupe 2 tandis que le plus faible DRT $(4,08 \pm 0,86)$ a été celui des accessions du groupe 9 . La plus forte valeur $(0,58 \pm 0,05)$ du rapport DRT/LgRT a été enregistrée au niveau des accessions du groupe 2 tandis que la plus faible valeur a été enregistrée au niveau des accessions des groupes $3(0,34 \pm 0,01), 8$ $(0,34 \pm 0,03)$ et $9(0,34 \pm 0,04)$. On peut donc déduire que les accessions des groupes 3,8 et 9 n'ont pas présenté une forme ronde (valeur éloignée de 0,5 ) selon Tarini et al. (2007). D'une façon générale, les différents paramètres agronomiques mesurés ont varié suivant les différents groupes d'accessions de PD étudiées. Ces résultats corroborent ceux de Djinet et al. (2015) qui dans leur étude comparée des paramètres agro-morphologiques de dix variétés de PD dans deux milieux réels (Tchad et Burkina Faso), ont également noté au niveau de ces variétés, une variabilité des paramètres (Rend, NbRT, PmRT, Bma, LgRT, DRT et LgRT/DRT) quelque soit le milieu d'étude. Les accessions des groupes 2 et 6 qui ont présenté des paramètres agronomiques intéressants peuvent être utilisées dans les programmes de sélection et d'amélioration de la PD.

Tolérance des accessions de patate douce à Cylas spp. : Les résultats ont indiqué que le pourcentage des racines tubéreuses infestées par Cylas spp. a varié de $0,00 \%$ à $36,74 \%$ selon les groupes d'accessions (Tableau 4). Le plus faible taux d'infestation $(0,00 \pm 0,00 \%)$ des racines tubéreuses a été enregistré au niveau du groupe 4 mais qui a donné le plus faible rendement $(2,40 \pm 1,71 \mathrm{t} / \mathrm{ha})$. La seule accession représentative de ce groupe est V37. Cette accession possède une particularité importante de par ses caractéristiques morphologique (péricarpe et pulpe de couleur orange) et nutritionnelle [riche en $\beta$-carotène, un précurseur de la vitamine $A$ (Laurie et al., 2012)]. Le plus fort taux d'infestation $(36,74 \pm 6,24 \%)$ des racines tubéreuses a été enregistré au niveau des accessions du groupe 8 caractérisé par un rendement moyen $(6,79 \pm 0,73 \mathrm{t}$ /ha) avec des racines tubéreuses à péricarpe et à pulpe de couleur crème. 
Tableau 3 : Paramètres agronomiques des groupes d'accessions de patate douce, pourcentage de racines tubéreuses infestées et indice de sévérité des dégâts causés par Cylas spp. sur les racines tubéreuses

\begin{tabular}{l|c|c|c|c|c|c|c}
\hline Groupe & LgRT & DRT & DRT/LgRT & NbRT & PmRT & Rend (t/ha) & Bma (t/ha) \\
\hline 1 & $16,49 \pm 0,93^{\mathrm{ab}}$ & $5,96 \pm 0,53^{\mathrm{bc}}$ & $0,39 \pm 0,02^{\mathrm{bc}}$ & $1,47 \pm 0,21^{\mathrm{bc}}$ & $253,49 \pm 34,45^{\mathrm{ab}}$ & $5,01 \pm 1,10^{\mathrm{bc}}$ & $12,67 \pm 1,30^{\mathrm{ab}}$ \\
2 & $14,95 \pm 1,11^{\mathrm{b}}$ & $8,36 \pm 0,86^{\mathrm{a}}$ & $0,58 \pm 0,05^{\mathrm{a}}$ & $2,77 \pm 0,43^{\mathrm{a}}$ & $299,07 \pm 69,72^{\mathrm{ab}}$ & $9,02 \pm 1,74^{\mathrm{ab}}$ & $12,12 \pm 1,99^{\mathrm{ab}}$ \\
3 & $16,67 \pm 1,00^{\mathrm{ab}}$ & $5,55 \pm 0,32^{\mathrm{bc}}$ & $0,34 \pm 0,01^{\mathrm{c}}$ & $1,78 \pm 0,29^{\mathrm{abc}}$ & $235,00 \pm 43,53^{\mathrm{ab}}$ & $4,68 \pm 0,84^{\mathrm{bc}}$ & $12,26 \pm 1,95^{\mathrm{ab}}$ \\
4 & $12,50 \pm 1,50^{\mathrm{b}}$ & $6,23 \pm 1,99^{\mathrm{abc}}$ & $0,52 \pm 0,06^{\mathrm{ab}}$ & $1,00 \pm 0,00^{\mathrm{c}}$ & $192,50 \pm 137,07^{\mathrm{ab}}$ & $2,40 \pm 1,71^{\mathrm{c}}$ & $5,72 \pm 1,63^{\mathrm{c}}$ \\
5 & $15,30 \pm 0,80^{\mathrm{b}}$ & $6,59 \pm 0,39^{\mathrm{ab}}$ & $0,45 \pm 0,02^{\mathrm{abc}}$ & $2,00 \pm 0,24^{\mathrm{abc}}$ & $335,40 \pm 41,57^{\mathrm{ab}}$ & $7,68 \pm 1,00^{\mathrm{abc}}$ & $8,12 \pm 0,89^{\mathrm{bc}}$ \\
6 & $16,86 \pm 0,61^{\mathrm{ab}}$ & $6,79 \pm 0,41^{\mathrm{ab}}$ & $0,44 \pm 0,01^{\mathrm{abc}}$ & $2,13 \pm 0,15^{\mathrm{ab}}$ & $443,20 \pm 53,30^{\mathrm{a}}$ & $10,31 \pm 0,97^{\mathrm{a}}$ & $10,30 \pm 0,90^{\mathrm{abc}}$ \\
7 & $12,94 \pm 1,00^{\mathrm{b}}$ & $6,96 \pm 0,85^{\mathrm{ab}}$ & $0,56 \pm 0,04^{\mathrm{a}}$ & $2,00 \pm 0,28^{\mathrm{abc}}$ & $344,44 \pm 109,03^{\mathrm{ab}}$ & $7,63 \pm 2,59^{\mathrm{abc}}$ & $14,20 \pm 2,96^{\mathrm{a}}$ \\
8 & $19,83 \pm 1,01^{\mathrm{a}}$ & $5,72 \pm 0,40^{\mathrm{bc}}$ & $0,34 \pm 0,03^{\mathrm{c}}$ & $2,52 \pm 0,20^{\mathrm{ab}}$ & $226,82 \pm 27,35^{\mathrm{ab}}$ & $6,79 \pm 0,73^{\mathrm{abc}}$ & $9,92 \pm 0,89^{\mathrm{abc}}$ \\
9 & $14,93 \pm 1,77^{\mathrm{b}}$ & $4,08 \pm 0,86^{\mathrm{c}}$ & $0,34 \pm 0,04^{\mathrm{c}}$ & $1,77 \pm 0,32^{\mathrm{abc}}$ & $133,33 \pm 42,49^{\mathrm{b}}$ & $2,77 \pm 0,96^{\mathrm{c}}$ & $11,27 \pm 2,03^{\mathrm{ab}}$ \\
F de Fisher & 3,89 & 2,50 & 4,88 & 2,54 & 3,11 & 3,96 & 0,91 \\
P(5\%) & $0,0003^{\star * *}$ & $0,0154^{*}$ & $0,0001^{* * *}$ & $0,0121^{*}$ & $0,0026^{* *}$ & $0,0002^{\star * *}$ & $0,0597^{\mathrm{ns}}$ \\
\hline
\end{tabular}

*** différence très hautement significative au seuil de $5 \%$; * différence hautement significative au seuil de $5 \%$ *différence significative au seuil de $5 \%$; ns : différence non significative au seuil de $5 \% ; F$ : variable de Fisher ; P (5\%) : probabilité au seuil de $5 \%$; LgRT : longueur des racines tubéreuses ; DRT : diamètre des racines tubéreuses; DRT/LgRT : rapport diamètre et longueur des racines tubéreuses ; NbRT: nombre moyen de racines tubéreuses par plant; Rend : rendement en racines tubéreuses; PmRT : poids moyen des racines tubéreuses; Bma: biomasse aérienne. Les moyennes suivies de la même lettre alphabétique dans une même colonne ne sont pas statistiquement différentes les unes des autres au seuil de $5 \%$.Les dégâts occasionnés par Cylas spp. sur les racines tubéreuses ont été plus importants sur les accessions du groupe $3(12,97 \pm 5,56)$ que sur celles du groupe $4(0,00 \pm 0,00)$. 
Tableau 4 : Paramètres de tolérance des accessions de patate douce face à Cylas spp.

\begin{tabular}{l|c|c|c}
\hline Groupe & Pourcentage d'infestation (\%) & Classe de résistance & Indice de sévérité \\
\hline 1 & $21,42 \pm 8,84^{\mathrm{ab}}$ & 2 & $8,92 \pm 4,32^{\mathrm{a}}$ \\
2 & $13,88 \pm 11,11^{\mathrm{ab}}$ & 2 & $4,16 \pm 2,94^{\mathrm{a}}$ \\
3 & $31,66 \pm 11,28^{\mathrm{ab}}$ & 3 & $12,97 \pm 5,56^{\mathrm{a}}$ \\
4 & $0,00 \pm 0,00^{\mathrm{b}}$ & 1 & $0,00 \pm 0,00^{\mathrm{a}}$ \\
5 & $18,58 \pm 6,49^{\mathrm{ab}}$ & 2 & $5,60 \pm 2,01^{\mathrm{a}}$ \\
6 & $25,98 \pm 4,50^{\mathrm{ab}}$ & 3 & $8,12 \pm 1,65^{\mathrm{a}}$ \\
7 & $27,77 \pm 14,69^{\mathrm{ab}}$ & 3 & $8,33 \pm 4,65^{\mathrm{a}}$ \\
8 & $36,74 \pm 6,24^{\mathrm{a}}$ & 3 & $11,37 \pm 2,21^{\mathrm{a}}$ \\
9 & $5,55 \pm 5,55^{\mathrm{ab}}$ & 2 & $1,38 \pm 1,38^{\mathrm{a}}$ \\
F de Fisher & 1,43 & - & 1,14 \\
$\mathrm{P}(5 \%)$ & $0,1873^{\mathrm{ns}}$ & - & $0,3363^{\mathrm{ns}}$ \\
\hline
\end{tabular}

1 : pas de dégâts apparents ( $0 \%$, très tolérant) $; 2$ : très peu de dégâts (1 à $25 \%$, tolérant) ; 3 : dégâts modérés ( 26 à $50 \%$, faible tolérance) ; 4 : dégâts considérables ( 51 à $75 \%$, sensible) ; 5 : dégâts sévères (76 à $100 \%$, très sensible). ns : différence non significative au seuil de $5 \%$. Les moyennes suivies de la même lettre alphabétique dans une même colonne ne sont pas statistiquement différentes les unes des autres au seuil de $5 \%$.

La sévérité des dégâts causés par Cylas spp. sur les racines tubéreuses est plus élevée au niveau des groupes d'accessions de PD ayant présenté les forts taux d'infestation de racines tubéreuses $(r=0,97)$. Cependant, ces paramètres indiquant le niveau de tolérance des accessions de patate douce à Cylas spp. sont fortement corrélés négativement avec les caractères CPPuRT, CSPuRT et DCSPuRT, tous liés à la pulpe des racines tubéreuses (Tableau 5). Ce qui signifie que la couleur de la pulpe de la racine tubéreuse influe sur l'infestation des racines tubéreuses par Cylas spp. A partir de cette étude, on peut retenir que les racines tubéreuses à pulpe crème et blanche sont plus susceptibles à l'attaque de Cylas spp. Par contre, les racines tubéreuses à pulpe orange sont plus tolérantes à Cylas spp. Les travaux d'Anonymous (2009) ont d'ailleurs montré que les génotypes de PD à pulpe orange possèdent une tolérance à Cylas spp. Le caractère de la couleur de la pulpe des racines tubéreuses devient ainsi un élément clé pour expliquer la disposition naturelle des accessions de PD à échapper à l'infestation par Cylas spp. Des analyses moléculaires pourraient aider à mieux identifier le déterminisme répulsif à Cylas spp. et existant au niveau des accessions de PD. Au vu des résultats obtenus, les accessions des groupes 2 et 6 ayant présenté des paramètres agronomiques intéressants et l'accession du groupe 4 ayant présenté une bonne tolérance à Cylas spp., peuvent être introduits dans un programme de sélection et d'amélioration variétale comme "parents" afin de produire des variétés génétiquement résistantes et à haut rendement pouvant être ainsi utilisées dans un programme de lutte contre contre Cylas spp. Cela permettra d'améliorer par la même occasion le revenu des producteurs. 
Tableau 5 : Corrélation entre les caractères du péricarpe, de la pulpe des racines tubéreuses, de leur pourcentage d'infestation et l'indice de sévérité des dégâts causés par Cylas spp.

\begin{tabular}{l|c|c|c|c|c|c}
\hline & CPPRT & CPPuRT & CSPuRT & DCSPuRT & $\begin{array}{l}\text { Indice de } \\
\text { sévérité }\end{array}$ & $\begin{array}{l}\text { Pourcentage } \\
\text { d'infestation }\end{array}$ \\
\hline CPPRT & -0.1266 & & & & & \\
CPPuRT & 0.7652 & & & & & \\
CSPuRT & 0.3946 & 0.3623 & & & & \\
& 0.3333 & 0.3778 & & & & \\
DCSPuRT & 0.0176 & 0.9679 & 0.4632 & & & \\
& 0.9671 & $<.0001$ & 0.2478 & & & \\
Indice de & 0.0856 & -0.7401 & -0.5925 & -0.7766 & & \\
sévérité & 0.8402 & 0.0358 & 0.1217 & 0.0234 & & \\
Pourcentage & 0.0338 & -0.7680 & -0.6433 & -0.8136 & 0.9718 & \\
d'infestation & 0.9367 & 0.0260 & 0.0852 & 0.0140 & $<.0001$ & \\
\hline
\end{tabular}

CPPRT : couleur prédominante du péricarpe des racines tubéreuses ; CPPURT : couleur prédominante de la pulpe des racines tubéreuses ; CSPuRT : couleur secondaire de la pulpe des racines tubéreuses ; DCSPuRT : distribution de la couleur secondaire de la pulpe des racines tubéreuses.

Les coefficients de corrélation sont les premières valeurs représentés dans le tableau. Les valeurs en dessous de ces coefficients représentent la probabilité.

\section{CONCLUSION ET APPLICATION DES RESULTATS :}

Des variabilités agro-morphologiques ont été enregistrées dans les différentes accessions de patate douce testées. II ressort de cette étude que les accessions de patate douce à péricarpe et à pulpe crème sont plus susceptibles à l'infestation par Cylas spp. tandis que les accessions à péricarpe et à pulpe orange, sont plus tolérantes à l'infestation par l'insecte. Les accessions des groupes 2 et 6 ont présenté de très bons paramètres agronomiques et celles du groupe 4 , un bon niveau de tolérance à Cylas spp. L'amélioration génétique de ces groupes d'accessions de patate douce par combinaison des caractères prévalant peuvent servir au programme de lutte contre Cylas spp. Cela peut consister à améliorer les niveaux de rendement des accessions du groupe 4 qui conservent déjà une tolérance à l'insecte, ou en améliorant les niveaux de tolérance à l'insecte, des accessions de patate douce des groupes 2 et 6 qui conservent déjà de bons rendements.

\section{REMERCIEMENTS}

Les auteurs de cet article remercient la Fondation Bill \& Melinda Gates qui a soutenu financièrement ce travail.

\section{RÉFÉRENCES BIBLIOGRAPHIQUES}

Agalè 0 . T. 2016. Utilisation des nématodes entomopathogènes et des accessions résistantes de patate douce contre Cylas puncticollis. Mémoire de Master en Gestion Durable des Nuisibles et Valorisation des Biopesticides. Faculté des Sciences Agronomiques, Université d'Abomey-Calavi, Benin, $62 \mathrm{p}$.

Amoatey H. M., Sossah F. L., Ahiakpa J. K., Quartey E. K., Appiah A. S., \& Segbefia M. M. 2016. Phenotypic profiles of different accessions of sweet potato (Ipomoea batatas L. Lam) in the coastal savanna agro-ecological zone of
Ghana. African Journal of Agricultural Research. 11(26), 2316-2328.

Andrade M.., Barker I., Cole D., Dapaah H., Elliott H., Fuentes S., Grüneberg W., Kapinga R., Kroschel J., Labarta R., Lemaga B., Loechl C., Low J., Lynam J., Mwanga R., Ortiz O., Oswald A., \& Thiele G. 2009. Unleashing the potential of sweetpotato in Sub-Saharan Africa: Current challenges and way forward. International Potato Center (CIP), Lima, Peru. Working Paper 2009-1. 197 p.

Anonymous, 2009. Biennial Report (2008 and 2009): $10^{\text {th }}$ Group meeting on Tuber Crops. In: Palaniswami M. S., \& Ramesh V. (eds.). All 
India Coordinated Research Project on Tuber Crops. India: Central Tuber Crops Research Institute, $193 \mathrm{p}$.

Bassey E. E., 2012. Field Evaluation of yield and resistances of local and improved sweet potato (Ipomoea batatas L. Lam) accessions to Cylas puncticollis and Meloidogyne incognita in Southeastern Nigeria. Asian Journal of Agricultural Science. 4, 390-394.

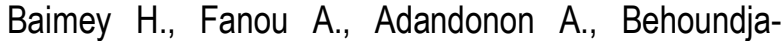
Kotoko O., Agandan N., Houssou G., \& Dossou-Agbede R. 2017. Sweet potato Ipomoea batatas (L.) storage practices used in southern Benin and the use of entomopathogenic nematodes to control sweet potato weevil (Cylas puncticollis Boheman) under laboratory conditions. Journal of Entomology and Zoology Studies. 5(6):549556.

CIPIAVRDC/IBPGR. 1991. Descripteurs pour la patate douce. Huamàn, Z. (ed.). International Board for Plant Genetic Resources, Rome, Italy. 51 $p$.

International Potato Center (CIP) (2013). Everything you ever wanted to know about Sweetpotato. Reaching Agents of Change ToT training manual. Topic 6: Sweetpotato Production and Management and Topic 7: Sweetpotato Pest and Disease Management. International Potato Center-Sub-Saharan Africa Regional Office (SSA). Nairobi, Kenya.

Djinet A. I., Nana R., Nguinambaye M. M., Badiel B., Konate B., Nanema L., Tamini Z. 2016. Etude du comportement de dix (10) variétés de patate douce cultivées à Bongor en saison sèche. International Journal of Innovation and Applied Studies. 17(4), 1384-1390.

FAOSTAT

http://www.fao.org/faostat/fr/\#data/QC

(2017).

Consulté le 29 Décembre 2017

Jackson D. M., Harrison H. F., \& Ryan Bohac J. R. 2012. Insect Resistance in Sweetpotato Plant Introduction Accessions. Journal of Economic Entomology. 105(2), 651-658.

Kagimbo F., Shimelis h., \& Sibiya J. 2017. Sweet Potato Weevil Damage, Production Constraints, and Variety Preferences in Western Tanzania: Farmers' Perception. Journal of Crop Improvement. 32(1), 107-123.

Karuri H. W., Ateka E. M., Amata R., Nyende A. B., Muigai A. W. T., Mwasame E., \& Gichuki S. T.,
2010. Evaluating diversity among Kenyan sweet potato genotypes using morphological and SSR markers. International Journal of Agriculture and Biology. 12, 33-38.

Koussao S., Gracen V., Asante I., Danquah E. Y., Ouedraogo J. T., Baptiste T. J., Belem J., \& Vianney T. M. 2014. Diversity analysis of sweet potato (Ipomoea batatas [L.] Lam) germplasm from Burkina Faso using morphological and simple sequence repeats markers. African Journal of Biotechnology. 13(6), 729-742.

Laurie S. M., Faber M., van Jaarsveld P. J., Laurie R. N., du Plooy C. P., \& Modisane P. C. 2012. $\beta$ Carotene yield and productivity of orangefleshed sweet potato (Ipomoea batatas L. Lam.) as influenced by irrigation and fertilizer application treatments. Scientia Horticulturae. 142, 180-184.

Muyinza H., Talwana H.L., Mwanga R.O. Stevenson P.C. 2012. Sweet potato weevil (Cylas spp.) resistance in African sweet potato germplasm. International Journal of Pest Management. 58, 73-81.

Okonya, J. S., \& Kroschel, J. 2013. Incidence, abundance and damage by the sweet potato butterfly (Acraea acerata Hew. and the African sweet potato weevils (Cylas spp.) across an altitude gradient in Kabale district, Uganda. International Journal of AgriScience. 3(11), 814-824.

Smit N. E. J. M., Downham M. C. A., Laboke P. O., Hall D. R., \& Odongo B. 2001. Mass trapping of male of Cylas spp. with sex pheromones: a potential IPM component in sweet potato production in Uganda. Crop Protection. 20, 643-651.

Stathers, T., Carey, E., Mwanga, R., Njoku, J., Malinga, J., Njoku, A., Gibson, R., \& Namanda, S. (2013). Tout ce que vous avez toujours voulu savoir à propos de la patate douce : Atteindre les agents du changement, manuel de formation des formateurs (FdF) 4: Production et gestion de la patate douce; Gestion des ravageurs et des maladies de la patate douce. Centre International de la Pomme de Terre, Nairobi, Kenya, 4, 73p.

Stathers T.E., Rees D., Nyango A., Kiozya H., Mbilinyi L., Jeremiah S., Kabi S., \& Smit N. 2003. Sweetpotato infestation by Cylas spp. in East Africa: II. Investigating the role of roots 
Kotchofa et al., J. Appl. Biosci. 2019 Tolérance de quelques accessions de patate douce (Ipomoea batatas L.) cultivées au sud-Bénin aux charançons de la patate douce (Cylas spp.) sous infestation naturelle

characteristics. International Journal of Pest Management. 49(2), 141-146.

Tarini A., Somda J. C., Vebamba O., Yameogo K. M., \& Belem J. 2007. Amélioration des apports en Vitamine des femmes et des enfants par la production et la consommation des patates douces à chair orange au Burkina Faso HKI, Ouagadougou. 18p.

Varin D., Blanc S., \& Brevar J. (2009). La culture de la patate douce en Nouvelle-Calédonie [Sweet potato cultivation in New Caledonia]. Association Inter-provinciale de gestion des Centres Agricoles. Centre des Tubercules Tropicaux. AICA, 27p. 